\title{
Elliptic and magneto-elliptic instabilities
}

\author{
Wladimir Lyra ${ }^{1, a}$ \\ ${ }^{1}$ Jet Propulsion Laboratory, California Institute of Technology, 4800 Oak Grove Drive, Pasadena CA, 91109
}

\begin{abstract}
Vortices are the fundamental units of turbulent flow. Understanding their stability properties therefore provides fundamental insights on the nature of turbulence itself. In this contribution I briely review the phenomenological aspects of the instability of elliptic streamlines, in the hydro (elliptic instability) and hydromagnetic (magneto-elliptic instability) regimes. Vortex survival in disks is a balance between vortex destruction by these mechanisms, and vortex production by others, namely, the Rossby wave instability and the baroclinic instability.
\end{abstract}

\section{Introduction}

The first documented observation of non-laminar motion in fluids is present in the work of Leonardo da Vinci [1]. He called the phenomenon turbolenza, after the Latin word for swirl (turbo). Sketching the flow, he wrote

[...] the smallest eddies are almost numberless, and large things are rotated only by large eddies and not by small ones, and small things are turned by small eddies and large.

Though written around 1500, this passage reads surprisingly modern, containing the seeds of concepts such as power spectrum and locality of the cascade. It contains also the insight that vortices are the fundamental unit of turbulent flow. The stability of vortices is thus a problem of paramount importance in fluid mechanics. Unveiling the mechanism that renders them unstable should provide vital insights into the nature of turbulence itself. The instabilities of magnetized vortices should likewise provide a similar framework when it comes to MHD turbulence.

Phenomenologically, turbulence can be described as a series of bifurcations, starting with a primary instability that converts shear into vorticity, creating vortices. This is followed by another bifurcation, a secondary instability, to break these vortices into lesser vortical structures. These in turn shall experience a sequence of "inertial instabilities", leading to a cascade. Though the Kelvin-Helmholtz instability and the Rayleigh-Taylor instability are well established as examples of primary instabilities, the highly successful theory of the turbulent cascade put forth by Kolmogorov [2] rested on a heuristic picture of secondary instability, established by early experiments [3]. It was not until the 80 's $[4,5]$ that the elliptic instability was introduced as a mechanism for the secondary instability. A fluid in riding rotation supports a spectrum of stable inertial waves, the simplest case being circularly

\footnotetext{
ae-mail: wlyra@jpl.nasa.gov
} 


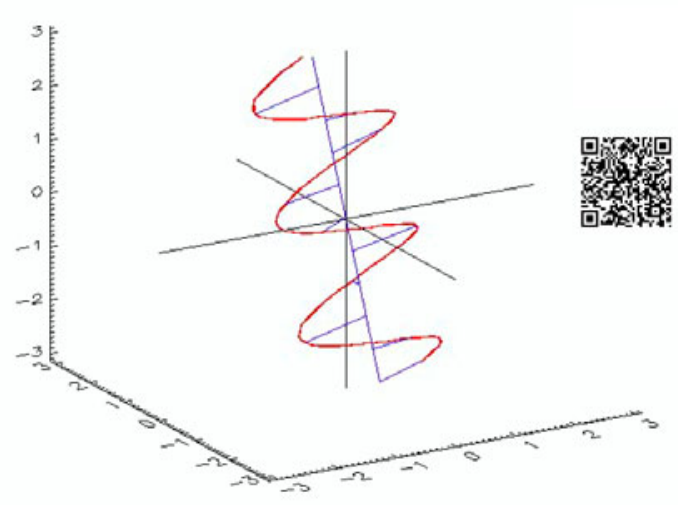

Figure 1. Illustration of the circularly polarized tilted Kelvin wave, a mode supported by fluid in uniform rotation. The contour in red traces the wave. As the wave is transverse, fluid parcels execute motion along the blue "arms" of the figure, oscillating both in-plane and vertically. Scan the barcode for an animation of the wave.

polarized transverse plane waves oscillating at twice the frequency of the base flow [6]. Strain is introduced when the streamlines pass from circular to elliptical, and some modes find resonance with the strain field, leading to de-stabilization.

The cascade reversal from inverse to direct when passing from $2 \mathrm{D}$ to $3 \mathrm{D}$ is a result of the different properties of $2 \mathrm{D}$ and $3 \mathrm{D}$ vortices. Two-dimensional vortices do not decay, merging viscously and growing to the integral scale; three-dimensional vortices also merge viscously, but before that they generally fall prey to the elliptic instability, which does not exist in two dimensions. Vortex survival depends on a balance between production and destruction.

\section{Elliptic instability}

A column of fluid under rigid rotation supports stable oscillations in the form of circularly polarized transverse plane waves (Fig. 1). Restored by the Coriolis force and propagating along a waveguide, these waves have also been called Kelvin waves. As the waves are transverse, if the direction of propagation coincides with the rotation axis, the action of the wave is that fluid parcels will execute in-plane epicyclic oscillations. The propagation vector may also have an angle $\theta$ with the rotation axis, in which case the fluid motion is no longer in-plane, executing both epicyclic and vertical motions, well known in galactic dynamics [7]. Destabilization occurs when strain in introduced; steepening gradients and providing a source of free energy. Instability occurs when a mode or pair of modes find resonance with the rotating strain field, which is to say when a multiple of the rotation frequency matches the frequency of the inertial waves.

The elliptic motion $U=\Omega[-(1-\varepsilon) y,(1-\varepsilon) x]$, where $0 \leq \varepsilon \leq 1$ is the ellipticity, is readily decomposed $U=\Omega(R+S)$ into rigid rotation $R=[-y, x]$ and the strain field $S=-\varepsilon[y, x]$. The growth rates, reproduced from [8], are shown in Fig. 2 in the $\chi-\theta$ plane. The aspect ratio $\chi$ of the streamlines is a measure of the strain. As the instability grows the vortex coherence is destroyed, energy cascades forward and dissipates; the flow relaminarizes.

As seen in Fig. 2, the instability is elliptical: there is no growth for $\chi=1$, i.e., circular streamlines in rigid rotation. The instability is also inherently three-dimensional: there is no growth for $\theta=0$, i.e., in-plane fluid oscillations. This later behavior is at the heart of the difference between the cascade in 2D and 3D. A stirring or primary instability, such as Rayleigh-Taylor or Kelvin-Helmholtz, generates the first eddies. The elliptic instability generates 3D turbulence out of this $2 \mathrm{D}$ motion, breaking the eddies. The growth rates are of the order of the turnover frequency, which explains why vortices have lifetimes of this order. As elliptic destruction occurs faster than viscous merging, the $3 \mathrm{D}$ cascade is direct. In two dimensions, there is no elliptic instability, and the eddies simply merge viscously. 

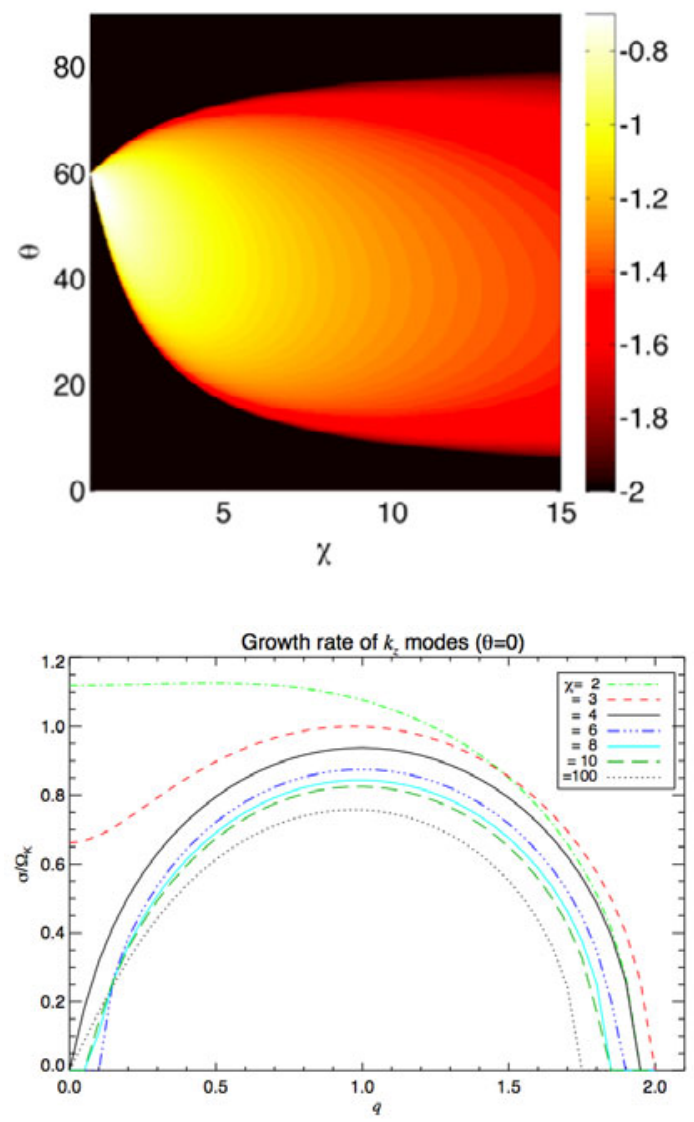

Figure 2. Growth rates for perturbations in closed elliptic streamlines, in the simplest case of absent background rotation or magnetic fields. In the $x$-axis $\chi$ is the aspect ratio of the base vortex, and $\theta$ the angle between the propagation vector of the inertial wave and the rotation axis of the vortex. There is no instability for circular streamlines $(\chi=1)$ : the instability is elliptic. No instability exists either for $\theta=0$, i.e., in-plane motion. The elliptic instability is inherently three-dimensional. Color-coded is the logarithm of the growth rate. Reproduced from [8].

Figure 3. Growth rates of the horizontal $(\theta=0)$ mode of the magneto-elliptic instability in the presence of rotation, for different aspect ratios of the base vortex. The quantity in the $x$-axis is $q=k / k_{\mathrm{BH}}$, where $k_{\mathrm{BH}}$ is the Balbus-Hawley wavenumber. As $\chi \gg 1$ we approach the pure shear limit $(\varepsilon=1)$ and the MRI growth rate is recovered: the MRI is a limiting case of the more general magneto-elliptic-rotational instability. Notice that for $q=0$ (no magnetic field), there is growth for $\chi=2$ and $\chi=3$, recovering the result of Rayleigh instability of horizontal modes of the hydro elliptic instability in the presence of rotation. Reproduced from [12].

Adding a background rotation has significant effects for the stability. The system now has two timescales: the turnover vortex time and that of the background flow. Not only that, the motions can be either aligned (cyclonic vortex) or anti-aligned (anti-cyclonic). Not surprisingly, the strongest instability occurs for anticyclonic motion, as the two flows rotating in opposite directions greatly enhances the effective shear on a fluid parcel. In this case, the in-plane, horizontal, motion gets de-stabilized. This instability is not of resonant nature, but centrifugal, appearing as exponential growth of epicyclic disturbances. This behaviour invites a connection with the Rayleigh (centrifugal) instability, and indeed the mechanism is similar [8], suggesting that the Rayleigh instability is a limit of the elliptic instability in the presence of rotation.

\section{Magneto-Elliptic Instability}

When magnetic fields are introduced in the problem, the addition of Alfvén waves enriches the families of unstable modes. In the absence of rotation, the magneto-elliptic instability is a parametric instability as well [9], have three unstable branches in the $\chi-\theta$ plane. The first two are "hydrodynamic" and "magnetic", corresponding to resonant destabilization of Kelvin and Alfvén waves, respectively. A third, "mixed" mode occurs through instability of a pair of modes when one is hydrodynamic and the other is magnetic, or a Kelvin-Alfvén instability. 

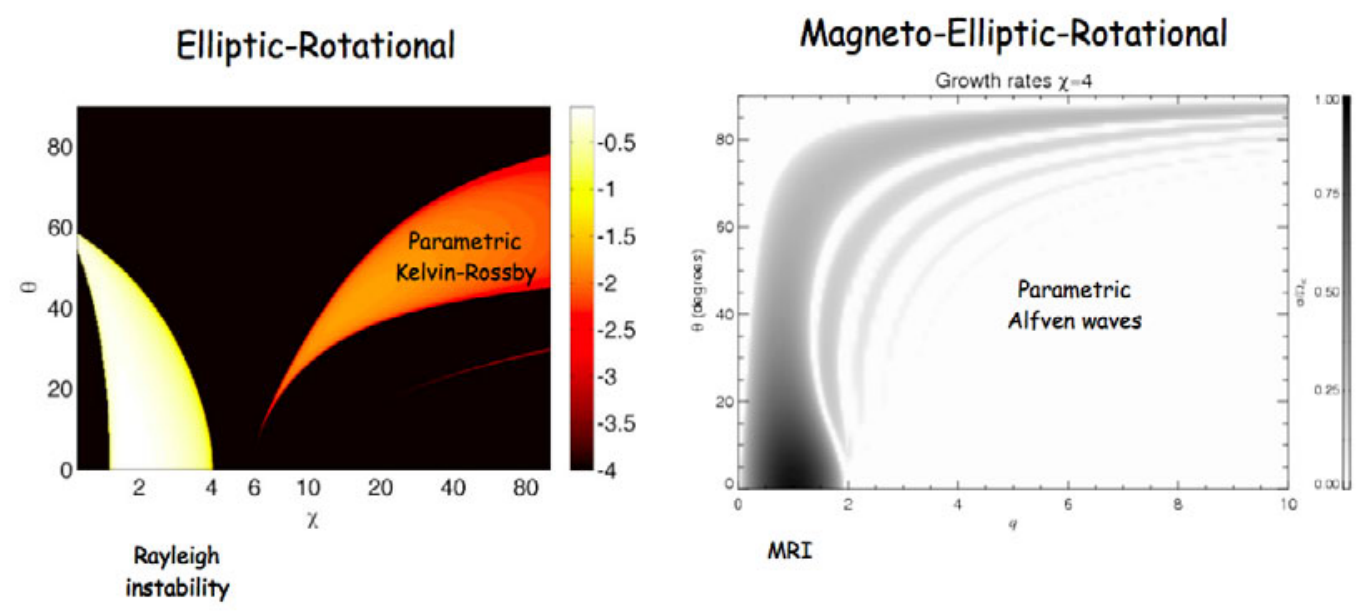

Figure 4. The four identified modes of vortex destruction. In the hydro case (left panel, reproduced from [8]), the Rayleigh instability of horizontal modes, and the parametric resonant tuning of 3D inertial waves with the underlying strain field. In the magnetic case (right panel, reproduced from [12]), the magneto-rotational instability of horizontal modes, and resonant tuning of Alfvén waves. These processes tend to oppose vortex coherence, as growing perturbations extract kinetic energy from the vortical motion. Vorticity is scattered, cascading forward, and eventually being removed by viscosity. They thus behave as vorticity sinks. A vortex survives if there is a mechanism to counteract these losses, by injecting vorticity.

When background rotation is introduced, the same as had occurred to the elliptic instability ensues. When this background rotation runs opposite to the rotation of the vortex (anti-cyclonic motion), a fluid parcel becomes subject to an intense effective shear. Since the magnetic tension resists shear, leading to instability [10] a powerful unstable in-plane mode appears [11]. This instability is of course the magneto-rotational instability, in generalized form. Indeed, the dispersion relation of this horizontal mode reduces to that of the MRI in the pure shear limit of $\varepsilon=1$ (Fig. 4, [12, 13]). This provides an interesting unification, explaining the magneto-elliptic and magneto-rotational instabilities as different manifestations of the same magneto-elliptic-rotational instability.

\section{Formation and destruction of vortices in disks}

Four ways of destroying vortices in disks are identified in Fig. 4. In the non-magnetic case (EI), these are the Rayleigh instability for the horizontal mode, and the resonant destabilization of Kelvin waves. In the magnetic case (MEI), these are the MRI for the horizontal modes, and resonant destabilization of Alfvén waves.

Counterbalancing these there are two mechanics to inject vorticity in the flow. These are the Rossby wave instability (RWI, [14-19]), and the Baroclinic Instability (BI, [12, 20-22]). The former is a linear instability powered by a modification of the shear profile, that behaves as an external reservoir of vorticity. The latter is a nonlinear instability powered by buoyancy and thermal diffusion, that establish a nonzero baroclinic source term. 


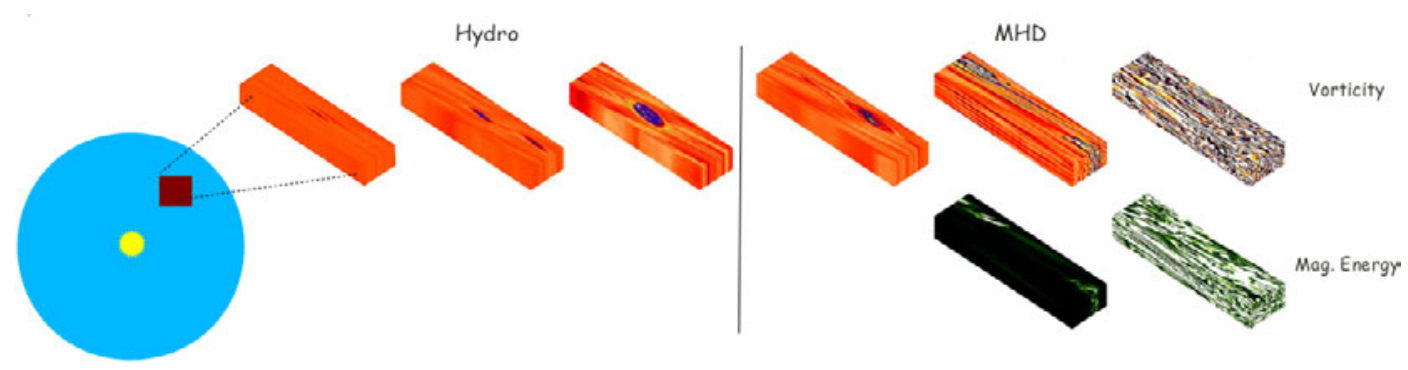

Figure 5. In non-magnetized flows, the vorticity injected by the baroclinic instability is able to counteract the vorticity lost the elliptic instability, at least the parametric, non-horizontal, version. In the magnetized case, however, the MEI growing inside the vortex core is more than the vortex can withstand. The conclusion is that baroclinic vortices are restricted to the dead zones of accretion disks.

As long as these vorticity sources (RWI and BI) are more powerful than the vorticity sinks (EI and MEI), a vortex can survive. It has been shown in the literature [12, 22] that despite the elliptic instability, the baroclinic instability keeps 3D vortices coherent. The result is core turbulence only, as the vorticity lost by the EI is replenished by that injected by the BI.

It has also been shown [12] that the same is not true for the MEI, with a magnetized baroclinic vortex getting quickly destroyed by the strong excitation of magneto-elliptic modes Fig. 5. Yet, magnetized vortices are seen in global, high-resolution simulations [19]. These were triggered by the RWI, albeit artificially, due to an intense peak in magnetic pressure in the initial condition, leading to a non-Keplerian shear profile in the active zone. The cylindrical simulation was performed at very high resolution, $N_{r}, N_{\phi}, N_{z}=(768,1536,128){ }^{1}$ ranging $\pi$ in azimuth and 0.4 to 2.5 in radius. This translates into 40 grid points per scale height, more than enough to resolve the unstable magnetoelliptic modes. So, either the said phenomenon is not a vortex but a zonal flow, or the RWI provides vorticity faster than the MEI can destroy it, as is the case with the BI and EI. If that is the case, we can group the processes in order of strength as $\mathrm{EI}<\mathrm{BI}<\mathrm{MEI}<\mathrm{RWI}$.

\section{References}

[1] da Vinci, L. ca 1500, in The notebooks of Leonardo da Vinci, Volume 2, Jean Paul Richter editor 1880, Dover Publications, Jun 1, 1970.

[2] Kolmogorov, A., Proceedings for the USSR Academy of Sciences 30, 301-305 (1941)

[3] Taylor, G. I., Phil. Trans. R. Soc. Lond. 223, 289-343 (1923)

[4] Pierrehumbert, R. T., Phys. Rev. Lett. 57 (17), 2157-2159 (1986)

[5] Bayly, B. J., Phys. Rev. Lett. 57 (17), 2160-2163

[6] Chandrasekhar, S., Hydrodynamic and hydromagnetic stability, Courier Dover Publications, 1961

[7] Binney, J. J. \& Tremaine, S. Galactic Dynamics, Princeton University Press, 1987

[8] Lesur, G. \& Papaloizou J. C. B., Astron. \& Astrophys. 498, 1-12 (2009)

[9] Lebovitz, N. R., \& Zweibel, E., Astrophys. J. 609, 301-312 (2004)

[10] Balbus, S. A. \& Hawley J. F., Astrophys. J. 376, 214-222 (1991)

\footnotetext{
${ }^{1}$ It also made use of high-end computing, being calculated in over 18000 processors at the NICS-Kraken cluster. We used the Pencil Code, with which we achieve nearly linear scalability in NICS-Kraken, up to 70000 processors.
} 
[11] Mizerski, K. A. \& Bajer, K., J. Fluid. Mech. 632, 401-430 (2009)

[12] Lyra, W. \& Klahr H., Astron. Astrophys. 527 138-157 (2011)

[13] Mizerski, K. A., \& Lyra, W. J. Fluid. Mech. 698, 358-373 (2012)

[14] Papaloizou, J. C. B. \& Pringle, J. E., Mon. Not. R. Astron. Soc. 208, 721-750 (1984)

[15] Lovelace, R. V. E., Li, H., Colgate, S. A., \& Nelson, A. F., Astrophys. J. 513, 805-810 (1999)

[16] Lyra, W., Johansen, A., Klahr, H., \& Piskunov, N., Astron. Astrophys. 491, L41-L44 (2008)

[17] Meheut, H., Casse, F., Varniere, P., Tagger, M., Astron. Astrophys. 516, 31-39 (2010)

[18] Lin, M.-K., Astrophys. J. 754, 21-36 (2012)

[19] Lyra, W. \& Mac Low, M.-M., Astrophys. J. 756, 62-71 (2012)

[20] Klahr H., Astrophys. J. 606, 1070-1082 (2004)

[21] Petersen, M. R., Stewart, G. R., \& Julien, K., Astrophys. J. 658, 1252-1263 (2007)

[22] Lesur, G. \& Papaloizou, J. C. B., Astron. Astrophys 513, 60-71 (2010) 